\title{
Los procesos de distritación electoral y el uso del criterio de comunidad de interés*
}

\author{
Carlos J. Vilalta Perdomo**
}

Una adecuada distritación electoral es elemento indispensable para el justo ejercicio democrático. No obstante, en México no se ha puesto aún suficiente atención en este tema. Este trabajo ofrece una contribución metodológica al centrarse principalmente en el criterio de "comunidad de interés". Los resultados de un ejercicio experimental de distritación electoral para el Estado de México demuestran que la utilización de este criterio bajo una dimensión metropolitana, junto con un nuevo algoritmo y el uso indistinto de diversas unidades geográficas al momento de redistritar, ofrece buenos resultados numéricos. De manera accesoria, se coincide con la opinión generalizada de que el proceso de redistritación oficial de 1996 representó un avance sustancial respecto a la de 1978, pero también se sugiere que ésta es perfectible y se puede avanzar más en la aplicación de nuevos criterios rectores en posteriores redistritaciones.

Palabras clave: distritos electorales, redistritación electoral, regionalización política, México.

Fecha de recepción: 6 de septiembre de 2000.

Fecha de aceptación: 9 de marzo de 2001.

\section{Introducción}

El estudio de los procesos de distritación es un tema fundamental para los científicos sociales interesados en la representación política. México cuenta desde 1996 con una nueva geografía electoral federal, la cual vino a sustituir a la de 1978, cuya modificación era necesaria al haber cambiado considerablemente la dinámica demográfica del país durante esos 18 años. Esta nueva distritación respondió al objetivo político de avanzar en la reforma electoral, para que las elecciones al Congreso $\mathrm{Fe}$ deral de 1997 se llevaran a cabo de una manera apropiada.

El estudio de los distritos electorales puede ser abordado desde diversos ángulos. Unos trabajos se refieren a las ventajas y desventajas

\footnotetext{
* Este trabajo forma parte de la tesis presentada en 1998 para obtener el grado de maestro en Estudios Urbanos por El Colegio de México. El autor agradece los valiosos comentarios de los profesores Gustavo Garza y Crescencio Ruiz, la invaluable ayuda de Jaime Ramirez del Laboratorio de Sistemas de Información Geografica de El Colegio de México y los comentarios de dos dictaminadores. Invariablemente, el autor es responsable por cualquier error $\mathbf{u}$ omisión.

** Instituto Tecnológico y de Estudios Superiores de Monterrey. Campus Ciudad de México. Correo electrónico: vilalta@mail.pdx.edu
} 
de contar con tales distritos, mientras que otros se dedican a analizar el comportamiento electoral en los mismos o bien se concentran en examinar el procedimiento en que son delimitados. El presente pertenece a este último tipo. Muy pocos trabajos en el país se ocupan de la parte técnica de la geografía electoral. Como dice Lujambio (1987), uno de los temas más olvidados en el estudio de las elecciones mexicanas es el grado de representatividad de la población, y en este sentido, de la distribución poblacional entre los distritos electorales.

Un proceso de distritación es un ejercicio de regionalización política complejo, pero considerando que acepta correcciones (Pacheco, Becerra y Woldenberg, 1997), en este trabajo se presenta un procedimiento alternativo al empleado en la redistritación oficial de 1996. Se muestran los resultados de un ejercicio técnico de regionalización electoral que logra reducir considerablemente las diferencias poblacionales entre distritos y que sigue al mismo tiempo el cumplimiento del criterio de la "integridad de comunidades" desde una perspectiva urbano-metropolitana.

\section{El distrito electoral}

El distrito electoral es una división del territorio al interior de cuyos límites se desarrolla un hecho político; "una actividad que aglutina a un conjunto de personas que se organizan para intervenir socialmente, a fin y efecto de alcanzar sus intereses" (Sánchez, 1992).

Entre las definiciones representativas de "distritos electorales" destacan las siguientes:

- "Área geográfica representada por un miembro del Congreso" (Elliot y Ali, 1984).

- "Áreas que mandan a los representantes al Parlamento, y cuya naturaleza (tamaño, etc.) está determinada por la ley electoral" (Johnston, 1979).

- "Areas geográficas para elegir a los representantes de quienes en ellas viven ante el Poder Legislativo" (Comité Técnico para el Seguimiento y Evaluación de la Redistritación, CTSER, 1996) (IFE, 1996).

\section{El distrito electoral como espacio y región}

El distrito electoral puede ser visto como un espacio funcional. Para Julliard (Bourne y Simmons, 1978), el espacio no es sólo una repre- 
sentación física de áreas terrestres, sino un ámbito en donde se desarrollan eventos de todo tipo. De tal manera, podemos pensar en toda una variedad de espacios según el evento de que se trate. En este caso concreto, son el aspecto territorial por medio del cual la institución de la representación democrática toma forma. Esta representación está fundada en el principio de que el pueblo es el titular del poder político, y que al no poder ejercerlo directamente, por vivir en comunidades extensas, funcionalmente lo delega en sus representantes democráticamente electos.

Un distrito electoral es también un espacio político, ya que representa una delimitación espacial del poder del pueblo y en él se manifiestan relaciones de poder. Sólo una delimitación espacial.justa del poder del pueblo -o sea, una regionalización adecuada- puede dar respuesta legítima a un ejercicio democrático. Los principios elementales de justicia, libertad e igualdad que rigen actualmente en las sociedades democráticas sólo pueden ser llevados a la práctica si las relaciones de poder son equilibradas por los redistritadores (Gelman y King, 1994).

¿Cómo debe de ser un distrito electoral y qué proceso habrá de seguirse para que estos espacios político-funcionales sean justos? Para responder es necesario acudir a los principios elementales de la regionalización.

Nuevamente para Julliard (Bourne y Simmons, 1978), una región es siempre una forma de organización del espacio. En este sentido, se puede concebir a una región como un territorio definido por uno o más aspectos propios.

Un distrito electoral es una región -un espacio funcional delimitado- porque:

- Las personas siempre actúan sobre un territorio, y si lo desean, politizan su entorno. Convierten algunos asuntos en temas públicos, y para ello organizan una estructura político-administrativa.

- Al politizar su espacio, delimitan territorios para este efecto, logrando entonces una representación espacial del poder y una delimitación territorial para efectos de su ejercicio indirecto.

No hay una organización política que esté exenta, por lo menos idealmente, ni por mucho tiempo, del concepto de territorialidad. ${ }^{1}$

\footnotetext{
${ }^{1}$ Las circunstancias extraordinarias se presentan por ejemplo, en tiempos de guerra, con la instalación temporal de un Estado en territorio ajeno. Pero aun sin guerra,
} 
El territorio es un medio para la existencia de la interacción de una sociedad, y la regionalización política del mismo es un elemento sine qua non para la adecuada convivencia social (Sanguin, 1981).

Un proceso de regionalización -de clasificación- debe cumplir los siguientes principios (Grigg, 1967): ${ }^{2}$

- El propósito de la regionalización debe dirigir los criterios elegidos.

- Las regionalizaciones tienen un propósito específico, y raramente sirven a dos propósitos diferentes.

- Siempre existen diferencias de tipo entre los objetos a regionalizar. Los objetos que difieren entre ellos no son fácilmente conjugables.

- Las regionalizaciones no son absolutas. Siempre que se obtenga más conocimiento sobre los objetos a clasificar, deberán ser modificadas.

- Cuando se divide el grupo de objetos, la división debe ser exhaustiva y las clases formadas deben ser excluyentes.

En este sentido, el distrito electoral tiene un propósito específico, diferenciable, exclusivo, y su forma de delimitación es perfectible. Los valores elementales de la representación democrática están en convención con estos principios. El problema técnico radica en realizar una adecuada regionalización electoral.

\section{Características particulares de los distritos electorales}

Como vimos, regionalizar es una labor de clasificación. En consecuencia, los principios elementales de esta tarea se pueden aplicar en el momento de delimitar distritos. Sin embargo, es difícil llevar a cabo una clasificación de este tipo en materia electoral debido a:

- Las relaciones de poder que intervienen en el proceso de delimitación; y

- la misma complejidad del procedimiento, que depende de los criterios elegidos.

Este trabajo aborda el segundo tipo de complejidad: la dificultad técnica. Ciertos aspectos distinguen a los distritos electorales de los

se puede presentar el de territorialidad como concepto de unión social. En la diáspora judía, el regreso a la tierra prometida tenía un fuerte sentimiento de territorialidad.

${ }^{2}$ Ésta es una síntesis de los principios de regionalización ofrecidos por Grigg. 
otros tipos de regiones políticas -municipios, estados, etc.-, y esto los hace dificiles de conjuntar en el proceso.

La primera dificultad se presenta desde el momento en que los distritos electorales tienen como noción y requisito esencial la igualdad, que debe serlo en relación con el valor de cada voto. ${ }^{3}$

Técnicamente hablando, el criterio de igualdad poblacional debería denominarse "límite de desviación a la media poblacional". En teoría, cada distrito debe tener la misma población, pero siendo esto técnicamente imposible, el procedimiento aceptado es acercarse lo más posible al promedio ideal, definiendo un límite diferencial. ¿Pero qué tanta diferencia se puede o se debe aceptar? Al respecto hay diversas opiniones sobre qué tanta desviación poblacional a la media distrital es aceptable. Las cifras que se manejan varían entre 2 y 15\%. La Suprema Corte de Estados Unidos, en Mahan $v s$. Howell, deliberando sobre un proceso de redistritación en el estado de Virginia, aceptó hasta una desviación de $16.4 \%$, ya que conservó la mayoría de las subdivisiones políticas, colocando como esencial en este caso el criterio de "comunidad de interés" (Dixon, en Grofman et al., 1982).

La distritación mexicana de 1996 siguió en su proceso la regla de $15 \%$ por arriba o abajo de la media distrital. Es obvio que cuan to más grandes sean, poblacionalmente hablando, las unidades geográficas con las que se distrita -por ejemplo municipios-, más difícil será ajustarse al criterio igualdad poblacional. $\mathrm{Al}$ respecto es interesante ver que en Estados Unidos las decisiones de la Corte Suprema han hecho disminuir la importancia de criterios como "compacidad" frente a otros como "representación minoritaria" y "cero diferencia poblacional"; "la forma en que se ve no lo es todo, cuando se trata de entidades políticas" (Donovan, 1991).

En la práctica, cuando se intenta lograr una perfección en la igualdad poblacional, se tiende a reducir la compacidad de los distritos (Backstrom, en Grofman et al., 1982). Sin una forma de crecimiento predeterminada e imparcial, la simple pretensión de igualdad poblacional puede ocasionar una manipulación de los límites distritales a fin de incluir o excluir votantes a favor de cierto partido político. Tal es el riesgo de gerrymandering: Dispersar a la oposición y concentrar partidarios en el territorio (Ostdiek, 1995).

3 "Si no son iguales, no tienen las mismas cosas; de ahî las luchas y los conflictos, cuando los iguales no tienen ni se reparten iguales cosas..." (Aristóteles, 1985). 
Ahora bien, así como se intenta que todos los distritos tengan una población total lo más similar posible, también se tiende a considerar otros atributos compartidos y que constituyen los principios básicos de todo ejercicio de regionalización electoral o distritación. Estos principios son "contigüidad", "compacidad" y "comunidad de interés".

La regla de que un distrito electoral sea contiguo, o dicho de otra manera, que no esté separado en partes, ha sido aceptada universalmente (Dixon, en Grofman et al., 1982). Esto no representa un inconveniente para el cumplimiento de los otros criterios básicos. Cabe mencionar que este criterio es privativo de los distritos electorales, ya que otras unidades geográficas, por ejemplo los municipios, pueden prescindir de tal requisito.

Por razones lógicas, este criterio es el menos cuestionado y el más fácil de adoptar, ya que no se puede hacer un juicio valorativo sobre si un distrito es más o menos contiguo que otro. Es una característica geográfica que cuando está presente no se puede mejorar, a diferencia de otras. ${ }^{4}$

Un distrito que goza de compacidad es aquel que mantiene distancias similares desde todos sus lados hacia el centro. Un argumento a favor de lograr que los distritos sean compactos es que aquellos que no tienen tal forma pueden levantar sospechas de gerrymandering (Orr, 1970). En este sentido, la compacidad puede ser una solución técnica para la potencial manipulación política del territorio.

El distrito más compacto sería aquel con forma de círculo. Sin embargo, es imposible dividir un mapa con círculos. Evidentemente, lo más compacto es lo que menos lados tiene, pero no hay fórmulas generalmente aceptadas para medir la compacidad, por lo que se suele dejar a una observación de sentido común sobre el mapa.

Para el proceso de distritación oficial de 1996, esto implicó la obtención de distritos lo más parecidos a polígonos regulares. Como no existe un requerimiento legal al respecto, su cumplimiento depende principalmente de las formas de crecimiento o agregación de unidades geográficas en el modelo de redistritación que se utilice.

El criterio de "comunidad de interés", también denominado "integridad de comunidades" responde a la idea de que los ciudadanos vo-

\footnotetext{
${ }^{4}$ Sin contigüidad no habría compacidad, y ésta, aunque de manera subjetiva, sí se puede medir y mejorar. El Consejo General del IFE consideró este requisito en su criterio séptimo de redistritacion.
} 
tan conforme a su identificación con ciertos intereses, como por ejemplo de clase social, de ocupación o profesión, rural vs. urbano, etc. En Reynolds vs. Sims (1964), la Suprema Corte de Estados Unidos aceptó que unidades administrativas enteras fueran contenidas dentro de distritos electorales hasta donde la igualdad poblacional lo permitiera; "una redistritación que no atiende a las subdivisiones políticas o a las diferencias en el paisaje natural o histórico, puede ser algo más que una franca invitación al 'gerrymandering' (Warren, en Reynolds vs. Sims, 1964, citado por Dixon en Grofman et al., 1982). En este mismo sentido, en Taylor vs. McKeithen (1974) se admitió que las unidades políticas pueden significar mucho para los electores a través del tiempo y por lo mismo no deben dejar de considerarse al redistritar.

Una distritación que mantiene la integridad de unidades político administrativas y funcionales - v.g. integración urbana-, y que se desvía ligeramente de la igualdad poblacional, la cual nunca se puede alcanzar, es válida. Sin embargo, la integridad de comunidades no implica necesariamente una integridad social. Aquí precisamente entra una parte del debate en la ciencia regional cuando se discute sobre el centro y el límite de las regiones. Si se entiende que las regiones son homogéneas respecto de los criterios elegidos para diferenciarse, entonces también deben tener un límite o frontera. El punto que se discute es dónde está ese límite. Como Grigg (1967) comenta, también hay zonas intermedias, las cuales indistintamente podrían agregarse, en teoría, a cualquier región o distrito en este caso.

Es importante considerar que la integridad de las comunidades y el apego ciudadano a las divisiones político-administrativas son cosas diferentes, que la primera es cuestionable. Dentro de un municipio puede existir una completa segregación social.

En el debate sobre la homogeneidad o la heterogeneidad social de los distritos electorales, no queda claro si para conseguir una.justa representación, lo más adecuado es contar con distritos que tengan gente similar en cuanto a sus condiciones de vida, o distritos en donde coexistan grupos diferentes (Overby y Cosgrove, 1996). En este último caso, se ha recomendado respetar las proporciones nacionales o estatales de estos grupos. Por ejemplo, si la población rural en el país es $30 \%$ y la urbana $70 \%$, se procurará que cada distrito tienda a ese porcentaje (Backstrom, en Grofman et al., 1982). En Estados Unidos la noción de "comunidad de interés" es fundamental, porque se refiere a distinguir a la población por minorías y mayorías y a esto se le ha considerado un affirmative gerrymandering. 
Para la redistritación oficial de 1996, el criterio 5 del Consejo General del IFE hace una referencia directa a la integridad de comunidades, en particular cuando dice que "la distribución de los distritos se efectuará... abarcando preferentemente, en forma completa, pueblos, barrios, colonias, comunidades indigenas...”. Los criterios 4 y 6 del mismo Consejo hacen una referencia indirecta a la integridad de las comunidades cuando estipulan una atención a los municipios al delimitar distritos.

Los principios teóricos comentados aquí están contenidos en el listado de criterios generales del IFE de 1996 y fueron considerados en el proceso. Efectivamente, la mayoría de los distritos electorales federales vigentes tiene como características el ser moderadamente similares en población, contiguos y relativamente compactos. Sobre integridad de comunidades no se puede afirmar lo mismo, precisamente por la ambigüedad que una definición de este tipo conlleva.

\section{Los criterios y el método utilizados en la distritación oficial de 1996}

Ya se comentó que el principio más importante en la delimitación de distritos electorales es la igualdad poblacional, el cual garantiza el principio fundamental de "una persona, un voto". También se mencionó que existen otros principios elementales derivados de diversas consideraciones de tipo social y geográfico. El proceso de redistritación oficial de 1996 siguió un conjunto de criterios generales establecidos por el Instituto Federal Electoral, los cuales se instituyeron en el hilo conductor del proceso y se mencionan a continuación.

Estos criterios fueron acordados el 23 de enero de 1996, cuando el Consejo General del IFE en su acuerdo primero, segundo párrafo, estipuló que

Para la formulación del proyecto [...] se habrán de tomar en consideración los siguientes criterios:

1) Ningún distrito electoral federal uninominal podrá comprender territorio de dos o más entidades federativas.

2) Para la determinación del número de distritos electorales federales uninominales que habrán de comprender cada entidad federativa, se aplicará la fórmula de distribución conocida como de St. Lagüe, tomando como base los resultados del Censo General de Población de 1990.

3) Para determinar los límites distritales al interior de cada entidad federativa, se utilizará un modelo heurístico. 
4) Con base en el criterio de equilibrio demográfico, se habrán de determinar aquellos municipios, además del Distrito Federal, que por sí solos puedan contener uno o más distritos electorales federales uninominales. 5) La distribución de los distritos se efectuará de norte a sur y de oeste a este, respetando en lo posible accidentes geográficos y obras viales de importancia, abarcando preferentemente en forma completa, pueblos, barrios, colonias, comunidades indígenas integradas con base en aspeetos socioculturales, etcétera.

6) Los distritos electorales que por su densidad poblacional deban comprender el territorio de más de un municipio, se constituirán preferentemente con municipios completos.

7) Deberá propiciarse la unidad geográfica de los distritos electorales.

8) Deberán considerarse la infraestructura de vías de comunicación y los tiempos de traslado de las secciones electorales a la cabecera distrital que se establezca.

9) En la delimitación de los distritos electorales se procurará obtener la mayor compacidad característica consistente en que el perímetro de los distritos adquiera una forma geométrica lo más cercana posible a un polígono regular.

10) El margen de variación de población de cada distrito con relación al cociente de distribución no podrá exceder, en principio de $+/-15 \%$ al interior de cada entidad federativa, tratándose como casos particulares aquellos que por razones geográfico-poblacionales excedan el rango de variación señalado.

11) Se respetará la distribución seccional vigente. No se prefijarán las cabeceras distritales, dejando la determinación de las mismas a la aplicación de criterios de mayor población, de vias de comunicación y de servicios públicos.

Para redistritar, se decidió utilizar un modelo heurístico desarrollado por el mismo IFE. Una vez que se reasignó el número de distritos electorales que le correspondían a cada entidad estatal, se aplicó este modelo heurístico mediante varias corridas por computadora. ${ }^{5}$ Cada corrida representaba una opción de distritación, y se hicieron 3000 corridas para cada entidad.

El modelo obtuvo distritos a partir de tres universos diferentes de unidades geográficas. En primer lugar, a partir de municipios completos que en atención a su población no podían constituir por sí solos un distrito electoral, y que por ende se agruparon con otros para

\footnotetext{
${ }^{5}$ Para una descripción más detallada del metodo utilizado en la reasignación de distritos electorales para cada entidad, revísese el informe "La redistritación electoral mexicana de 1996. Informe sobre los resultados de la redistritación que presenta el Comité Técnico para el Seguimiento y Evaluación de la Redistritación al Consejo General del Instituto Federal Electoral", Instituto Federal Electoral, 1996.
} 
CUADRO 1

México: Población y distribución de distritos electorales federales en 1978 y 1996

\begin{tabular}{|c|c|c|c|c|c|}
\hline $\begin{array}{l}\text { Entidad } \\
\text { federativa }\end{array}$ & $\begin{array}{c}\text { Población } \\
\text { en } \\
1979 *\end{array}$ & $\begin{array}{c}\text { Distritos } \\
\text { asignados e } \\
1978\end{array}$ & $\begin{array}{c}\text { Población } \\
\text { en } \\
1990\end{array}$ & $\begin{array}{c}\text { Distritos } \\
\text { asignados en } \\
1996\end{array}$ & $n$ \\
\hline Aguascalientes & 497101 & 2 & 719649 & 3 & 1 \\
\hline Baja California & 1362834 & 6 & 1660865 & 6 & 0 \\
\hline B.C.S. & 201640 & 2 & 317786 & 2 & 0 \\
\hline Campeche & 384455 & 2 & 535193 & 2 & 0 \\
\hline Chiapas & 2179549 & 9 & 3210491 & 12 & 3 \\
\hline Chihuahua & 2249873 & 10 & 2441866 & 9 & -1 \\
\hline Colima & 1573711 & 7 & 1972343 & 7 & 0 \\
\hline Coahuila & 358153 & 2 & 428508 & 2 & 0 \\
\hline Distrito Federal & 9191295 & 40 & 8235765 & 30 & -10 \\
\hline Durango & 1320306 & 6 & 1349369 & 5 & -1 \\
\hline Guanajuato & 3083046 & 13 & 3982643 & 15 & 2 \\
\hline Guerrero & 2246230 & 10 & 2620632 & 10 & 0 \\
\hline Hidalgo & 1509960 & 6 & 1888348 & 7 & 1 \\
\hline Jalisco & 4670841 & 20 & 5302707 & 19 & -1 \\
\hline México & 7851156 & 34 & 9815773 & 36 & 2 \\
\hline Michoacán & 3123846 & 13 & 3548197 & 13 & 0 \\
\hline Morelos & 939805 & 4 & 1195057 & 4 & 0 \\
\hline Nayarit & 788732 & 3 & 824649 & 3 & 0 \\
\hline Nuevo León & 2584195 & 11 & 3098742 & 11 & 0 \\
\hline Oaxaca & 2399597 & 10 & 3019554 & 11 & 1 \\
\hline Puebla & 3330971 & 14 & 4126121 & 15 & 1 \\
\hline Querétaro & 707938 & 3 & 1051242 & 4 & 1 \\
\hline Quintana Roo & 157288 & 2 & 493276 & 2 & 0 \\
\hline San Luis Potosí & 1752842 & 7 & 2003198 & 7 & 0 \\
\hline Sinaloa & 2010787 & 9 & 2204046 & 8 & -1 \\
\hline Sonora & 1620500 & 7 & 1823609 & 7 & 0 \\
\hline Tabasco & 1155313 & 5 & 1501738 & 6 & 1 \\
\hline Tamaulipas & 2078051 & 9 & 2249583 & 8 & -1 \\
\hline Tlaxcala & 524668 & 2 & 761260 & 3 & 1 \\
\hline Veracruz & 5328279 & 23 & 6228231 & 23 & 0 \\
\hline Yucatán & 1047611 & 4 & 1362944 & 5 & 1 \\
\hline Zacatecas & 1150531 & 5 & 1276311 & 5 & 0 \\
\hline Total & 69381104 & 300 & 81249696 & 300 & \\
\hline $\begin{array}{l}\text { Media nacional } \\
\text { distrital }\end{array}$ & 231270 & & 272174 & & \\
\hline
\end{tabular}

* La población considerada para 1979 es una proyección de la Secretaría de Programación y Presupuesto que se realizó tomando como base el Censo de Población de 1970.

Fuente: Lujambio (1987) e IFE (1996). 
este efecto (v.g. Acambay en el Estado de México, con 47524 habs.). Luego, a partir de aquellos que podían tener un distrito, o casi uno, y que por simpleza se prefirió mantenerlos como un distrito electoral (v.g. Atizapán de Zaragoza, también en el Estado de México, que pese a su población de 315196 habs. fue preferible mantenerlo como un municipio-distrito). Yen tercer lugar, a partir de secciones electorales en aquellos municipios que por sí solos podían poseer dos o más distritos electorales (v.g. Ecatepec al que con 1218129 habitantes le correspondieron cuatro distritos, o Naucalpan, al cual con 786535 habitantes se le asignaron tres).

La forma en que el modelo asignó los distritos fue mediante la selección de un primer municipio denominado "semilla", al cual se iban agregando unidades -municipios o secciones, según fuera el caso del universo que se tratara- hasta obtener una población lo más cercana al promedio distrital. Como semilla inicial en el proceso, se seleccionó el municipio o sección electoral que se encontrara más al norte de la entidad. En caso de haber dos o más municipios -o secciones electorales- con la misma latitud norte, se seleccionó aquel que tuviera en su perímetro el punto que estuviera más al oeste de la entidad. Hubieron tres formas de agregación (central, por mejor vecino y mixta) de unidades a esta semilla. ${ }^{6}$

Una vez delimitado un distrito a su menor diferencia poblacional a la media distrital, siguió la selección de otra semilla de una manera aleatoria entre todas las unidades que no pertenecieran al primer distrito ya delimitado; y así se continuó en forma aleatoria con todas las semillas del resto de los distritos.

Este proceso de redistritación intentó llevar a cabo la mejor distritación posible, por lo que tales métodos de crecimiento se desarrollaron 1000 veces cada uno, para cada entidad federativa. La mejor solución encontrada, o sea, la distritación con el menor número de diferencias poblacionales entre distritos electorales, fue la que se seleccionó y se denominó "primera versión" de la redistritación. Posteriormente, a ésta se le incorporaron otros criterios y se llegó a una segunda versión.

El modelo utilizado es adecuado si se considera que posee una forma de delimitación que cumple formalmente con los criterios es-

\footnotetext{
${ }^{6}$ Para una explicación más detallada de las formas de agregación, véase el documento mencionado en la nota anterior.
} 
CUADRO 2

Criterios utilizados en cada versión de la redistritación oficial de 1996

\begin{tabular}{lc}
\hline \multicolumn{1}{c}{ Primera versión } & \multicolumn{1}{c}{ Segunda versión } \\
\hline 1) Igualdad poblacional & 1) Accidentes geográficos \\
2) Contigüidad & 2) Vias de comunicación importantes \\
3) Respeto a fronteras estatales, & 3) Integridad (ocasionalmente) de \\
municipales y seccionales & pueblos, barrios y comunidades \\
4) Compacidad & \\
\hline
\end{tabular}

tablecidos. El hecho de haber dividido los municipios en universos diferentes, por razones de tamaño poblacional, es razonable e igualmente imparcial. Se encontró que para todo el país 42 municipios y 14 de las 16 delegaciones del Distrito Federal podrían contener uno o más distritos electorales. Se optó por separar a aquellos que redondeando por el método de St. Lagüe les correspondieran uno o más distritos. Se llegó a 14 municipios con un solo distrito electoral, 28 con dos o más, y el Distrito Federal se distritaría sin considerar las fronteras delegacionales, como una masa urbana compacta.

Para formar distritos, el IFE (1996) prefirió utilizar secciones electorales sobre las Áreas Geoestadísticas Básicas (AGEB) por los siguientes motivos: dades.

1) La Constitución no especifica que se deben utilizar estas uni-

2) El IFE estimó que las AGEB son herramientas operativas diseñadas para organizar el levantamiento censal y no para construir áreas geográficas; o sea, que estas unidades no fueron diseñadas para realizar una distribución distrital.

3) La cartografía del INEGI carece de precisión métrica.

4) El Censo General de 1990, sólo contiene datos para las AGEB urbanas, por lo que no es posible delimitar distritos al interior de municipios que no contienen este tipo de áreas.

5) Por otro lado, se acordó respetar -conforme al criterio undécimo del IFE- la división seccional vigente, y al no coincidir las secciones con las AGEB, habría que estimar la población de las secciones y de partes de ellas que se encuentran en los límites de los distritos conformados a partir de las AGEB, labor que se consideró poco confiable debido a la dificultad de hacer estimaciones de población de áreas geográficas pequeñas. 
Los motivos de la Junta General Ejecutiva del IFE con mayor fundamento para preferir a las secciones electorales sobre las AGEB son el 4 y el 5. Los motivos 1 a 3 son en realidad prescindibles. Por un lado, el argumento sobre la libertad constitucional no es trascendente. Por el otro, las unidades censales no imposibilitan de ninguna manera su utilización como unidades básicas de distritación. Es más, legalmente su uso se valida, ya que cumplen perfectamente con el mandato constitucional de utilizar el Censo de Población como fuente de información para distribuir los distritos. ${ }^{7}$ También la cartografía digital con que se contaba entonces era aceptablemente precisa para delimitar colonias y vías de comunicación dentro de las ciudades. Se debe agregar además que al decidirse a realizar la redistritación por secciones electorales para aquellos municipios con dos o más distritos electorales, tuvo que estimarse la población de cada sección, ya que en éstas se contabilizan los electores -ciudadanos empadronados, lógicamente mayores de edad-, y no la población total.

Otra ventaja que argumenta el IFE para preferir las secciones electorales sobre las AGEB es que aquéllas son una fuente continua de información. Por otro lado, siendo el padrón la fuente ante la cual se registran los ciudadanos para gestionar la credencial de elector -necesaria para muchos trámites de la vida cotidiana- la gente se ve más obligada a acudir a éste, y en comparación el censo puede presentar sesgos más grandes en el conteo. En este sentido, se argumenta que el nivel de cobertura del Padrón Electoral Federal era elevado.

En todo caso, las diferencias poblacionales entre los distritos considerados en el mapa electoral vigente, que es lo que califica mayormente una redistritación, no se debieron a la utilización de estas unidades sino a la distinción que se hizo de los municipios por el tamaño de su población. De hecho, las secciones electorales también se utilizaron para redistritar no sólo en el interior de los municipios, sino para toda la extensión del Distrito Federal. Aquí la utilización de este tipo de unidades no dejó dudas sobre su aceptable efectividad, ya que ninguno de los distritos del Distrito Federal está fuera del rango poblacional predefinido de $15 \%$ por encima o debajo de la media distrital.

\footnotetext{
${ }^{7}$ Artículo 53 de la Constitución Política de los Estados Unidos Mexicanos.
} 
Una propuesta de redistritación alternativa

La propuesta alternativa es principalmente una aplicación del principio de comunidad de interés, basado en las diferencias metropolitanas. Este principio indica que al momento de ir delimitando distritos, se deben atender aquellas diferencias socioeconómicas que se hallan entre pueblos, barrios, colonias, comunidades indígenas etc. Para este efecto precisamente, se redactó el criterio 5 del IFE que estableció que "La distribución de los distritos se efectuará... abarcando preferentemente, en forma completa, pueblos, barrios, colonias, comunidades indígenas integradas con base en aspectos socioculturales...". Otra referencia se encuentra en el mismo criterio 5, y es: "la distribución de distritos se efectuará... respetando en lo posible los accidentes geográficos y obras viales de importancia".

Nótese que este criterio oficial mantiene un buen grado de flexibilidad para hacer la división en distritos. Sobre la integridad de las comunidades, establece que será "preferentemente", y sobre los aspectos geográficos y las obras viales, indica "en lo posible". Esto significa que la "comunidad de interés" fue un criterio de importancia relativa, frente al de "igualdad poblacional", el cual constituyó el criterio principal en la regionalización electoral.

Efectivamente, el hecho de que en la "primera versión" de la distritación se haya hecho caso omiso a estas consideraciones alternativas, explica la importancia predominante de la variable "población". Sin embargo, en la "segunda versión" se aceptaron consideraciones de este tipo, y se hicieron modificaciones a la geografía electoral resultante de la "primera versión", atendiendo a las obras viales, la integridad de las colonias, etcétera.

La razón por la que estas variables quedaron relegadas a un segundo plano, fue que el método seguido en la redistrítación era al principio estrictamente poblacional. Se hizo una separación de municipios por la magnitud de su población, distinguiendo los que podían contener un distrito, los que podían tener dos o más, y los que por su tamaño reducido serían agrupados para formar distritos. Esta diferenciación es estrictamente numérica; no contiene ninguna clasificación por razones atribuibles al paisaje natural -accidentes geográficos-, ni de funcionalidad -obras viales-, ni sociales -comunidades diferentes por su nivel socioeconómico-. Una vez que ya había sido hecha la distritación, se consideraron estas variables y se modificó levemente la geografía obtenida. 
La propuesta alternativa estriba en hacer desde el principio una clasificación de municipios -como la unidad básica de distritacióncon base en sus características generales, que representan diferencias sociodemográficas entre las comunidades no metropolitanas (rurales y urbanas) y las metropolitanas.

Cuando se habla de lo "urbano" o de lo "rural", se hace referencia directa a porciones del terreno diferenciadas con base en la organización del espacio y en los paisajes distintivos. Los rasgos físicos del paisaje, la forma y fines de la organización territorial y las características socioeconómicas de los pobladores constituyen el conjun to de elementos que permiten calificar como urbano o rural un espacio. Estas características pueden ser consideradas en la delimitación de distritos electorales.

Las áreas urbanas y las rurales son visiblemente diferentes. Las primeras tienen un área de influencia heterogénea que en general es más grande que la rural. La pluralidad de actividades, la diversidad arquitectónica, la extensión de la superficie construida, y en general la alta densidad, distinguen estos tipos de áreas.

Las necesidades sociales en cada espacio son diferentes, por lo que sería democráticamente aceptable distinguir, al emprender un proceso de distritación, unidades geográficas diferentes, siempre y cuando sea posible. Por supuesto, no se piensa que la dicotomía urbano-rural sea la mejor manera de definir a la "comunidad de interés", pero sí constituye una buena aproximación para efectos de ejercicios de distritación electoral.

Queda claro que el criterio de "comunidad de interés" refuerza el cumplimien to estricto del principio de representatividad ciudadana. En este sentido, es viable hacer una distinción de distritos electorales metropolitanos y no metropolitanos (urbanos y rurales), ya que es una forma neutral y efectiva de obtener la representatividad de todos y de los diversos intereses. Las necesidades e intereses de la población dependen del ambiente, urbano o rural en que se desenvuelven, y suelen reflejarse en sus tendencias políticas y electorales. La diferenciación entre lo metropolitano y lo no metropolitano da un sentido de comunidades diversas con intereses también diferentes.

Lo que se quiere destacar aquí es que es preciso considerar los aspectos contrastantes entre las zonas metropolitanas y las no metropolitanas. Tanto iguales como desiguales tienen intereses nacionales y deben ser representados en el Congreso Federal, y es importante tener presente que el derecho a votar no garantiza automática ni siste- 
máticamente que todos somos iguales. Una distritación que no atienda adecuadamente al principio de comunidades de interés, no promoverá una:justa representatividad.

Por otro lado, no es la mejor solución social buscar únicamente la igualdad matemática al momento de delimitar distritos, pues cuanto más justa es la democracia mayor atención presta a las diferencias de la población, y más se preocupa por identificar grupos de interés para representarlos efectivamente. Una debilidad en los métodos de distritación es perseguir la igualdad poblacional como premisa única. Si no toma en cuenta las características la comunidad, la institución de la representación política es poco eficaz. No es gratuito que los procesos de redistritación en otros países atiendan con tanto detalle este principio.

La literatura especializada sugiere que metodológicamente cuanto más grande sea la cantidad de criterios que se consideran, más dificil será modificar arbitrariamente los límites de los distritos (gerrymandering). Esto es, los métodos más sencillos son también los más flexibles, y por ende los más alterables en beneficio de intereses particulares. Un algoritmo exhaustivo puede dar certidumbre al proceso de regionalización.

A continuación se presenta una explicación sucinta de los criterios que dirigieron la distritación experimental en este trabajo. En general, se agregaron nuevos elementos a los criterios generales establecidos por el IFE; algunos se modificaron levemente y otros se mantuvieron idénticos.

Los criterios que se agregaron y modificaron en el ejercicio fueron:

1) Con base en la diferencias urbano-rurales, llevadas a los niveles metropolitano y no metropolitano, se hará una diferencia entre los municipios de cada estado, con los cuales se llevará a cabo la distritación por grupos diferentes (no metropolitanos y metropolitanos).

2) La delimitación de los distritos se hará iniciando con una semilla la cual puede ser tanto un municipio que no llegue a rebasar la población media distrital como una AGEB que sea la más poblada. En este sentido, la forma de escoger las semillas será de las unidades geográficas más pobladas a las menos pobladas. Este procedimiento garantiza la imparcialidad que da el número de población total en cada área geográfica. ${ }^{8}$

\footnotetext{
${ }^{8} \mathrm{Si}$ bien hay varias maneras de escoger la semilla, nosotros preferimos esta por ser aleatoria, y principalmente porque sigue una secuencia centro-periferia que tiende a evitar las desigualdades que ocurren en los municipios o las AGEB que quedan aislados porque se adoptan criterios meramente geográficos, como es el caso de la unidad más al norte, utilizada en la distritación de 1996.
} 
3) El margen de diferencia poblacional entre cada distrito no podrá exceder $10 \%$ tratándose de distritos no metropolitanos y $8 \%$ en los metropolitanos. La razón de esta diferencia es que las unidades geográficas para distritar en cada universo son sumamente diferentes. Es más dificil mantener $8 \%$ en distritos conformados por municipios completos, que al ir agregando municipios o partes de éstos y las AGEB, indistintamente.

Habiendo ya explicado la inserción del criterio de comunidad de interés al nivel metropolitano, las unidades geográficas utilizadas (AGEB y municipios) y el algoritmo alternativo, describiremos a continuación los resultados obtenidos en el ejercicio experimental. Para este caso se utilizó el Estado de México, principalmente por haber sido la entidad más deficientemente redistritada en el proceso oficial de 1996.

\section{Resultados del ejercicio}

Vistas las dificultades prácticas de un proceso de distritación, se inicia este inciso en completo acuerdo con los tres supuestos del CTSER (IFE, 1996):

1) No existe la mejor distritación única.

2) No puede existir el método que genere tal distritación.

3) Sólo tiene sentido hablar de propuestas de distritación razonables.

El Estado de México era en 1990 la entidad con el mayor número de habitantes en la República (9 815795 ) y ocupa en la actualidad el primer lugar en la asignación de distritos, y por ende de diputados uninominales: 36. Se eligió esta entidad para el ejercicio experiomental por diversas razones. La primera es que presentaba, dentro de la distritación oficial de 1996, el mayor número de distritos fuera de rango (7, es decir, 19\%) frente a las demás entidades federativas. Esto implicaba un precedente de dificultad mayor para regionalizar, lo que la hacía una entidad interesante para experimentar estas ideas. $Y$ la segunda razón es que contiene en su interior tanto municipios de características rurales como otros de perfil metropolitano. En particular, posee municipios en dos zonas metropolitanas: Toluca y la Ciudad de México.

En el ejercicio, la forma en que se distinguieron los universos de municipios a partir de los cuales se iniciaría la distritación fue sencilla. En primer lugar, se siguieron las definiciones del INEGI en cuanto a zo- 
nas metropolitanas; el resto del estado no metropolitano se distritaría de manera conjunta. Entonces, había tres universos de municipios: el de la Zona Metropolitana de Toluca (ZMT) con 6, el de la Zona Metropolitana de la Ciudad de México (ZMCM) con 27, y el "resto del estado" con 89. Al realizar estas distinciones se encontró que quedaban tres "islas": una en el norte, otra al noreste y una más al sureste del estado. Estas islas son conjuntos de municipios no metropolitanos que quedaron aislados del resto de su universo por la forma física de la ZMCM. Estas islas no cuentan con población suficiente para conformar un distrito.

Este problema se solucionó de la manera siguiente: se pasaron municipios del universo de la ZMCM a esas islas del "resto del Estado de México" hasta contener la población suficiente para formar un distrito. Se tomó esa acción porque se necesitaba pasar menos municipios de la ZMCM para crear distritos electorales que viceversa. Por decirlo de otra manera, se siguió una regla económica de pasar de un universo al otro el menor número de unidades geográficas suficientes para conformar un distrito.

Una vez integradas estas islas, ya se tenían delimitados tres distritos electorales. El paso siguiente fue distritar el universo "resto del estado" (o no metropolitano). Aquí se fueron agregando, como se explicó en el inciso anterior, municipios con municipios. Una vez distritado este universo, se prosiguió con la ZMT, y finalmente la ZMCM. En estos dos casos se fueron creando distritos agregando indistintamente municipios con AGEB, tal y como el algoritmo alternativo lo indica. Esta labor se ejecutó de manera manual sobre el mapa.

Haciendo una comparación de los resultados de este ajercicio con la distritación oficial vigente, los resultados fueron los siguientes:

1) Se redujeron $204 \%$ las diferencias de población entre distritos frente a la distritación oficial.

2) Se mantuvo la contigüidad de todos los distritos. En la oficial, hay un distrito dividido en dos partes (distrito 16 en Tlalnepantla).

3) Sólo dos distritos estuvieron fuera del margen de aceptación adoptado de $10 \%$ y ninguno por arriba o abajo del límite oficial de 15 por ciento.

4) El promedio de las diferencias poblacionales a la media distrital fue de $2.33 \%$ en el caso del universo metropolitano, y de $5.83 \%$ en el no metropolitano. En total, la desviación promedio en el experimento fue de $3.20 \%$ mientras que en la oficial fue de 9.75 por ciento.

Desde el punto de vista puramente estadístico, se puede considerar que el ejercicio logró una mejor distritación. Respecto a la forma 
final de los distritos, la compacidad fue la parte menos elaborada (véase los mapas). El motivo es el algoritmo utilizado, el cual pone más interés en los criterios de igualdad poblacional y comunidad de interés que en la forma física del distrito electoral.

Sin embargo, considerando que el principio más importante es el de la igualdad poblacional, dentro de un esquema de imparcialidad la forma es lo que menos cuenta. Esto es, un distrito no compacto no implica una afectación de la representatividad electoral, como lo haría un distrito desigual en cuanto a la población representada, o un distrito separado por partes, es decir, no contiguo.

MAPA 1

Redistritación experimental del Estado de México

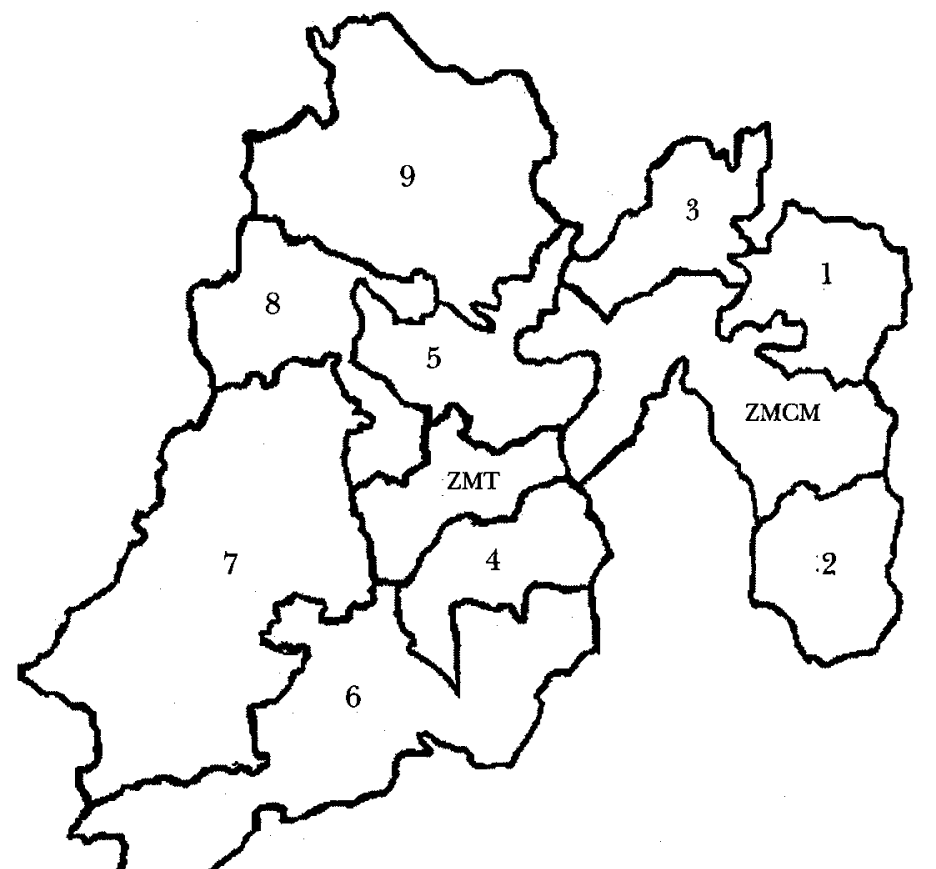


MAPA 2

Redistritación experimental de la Zona Metropolitana de Toluca

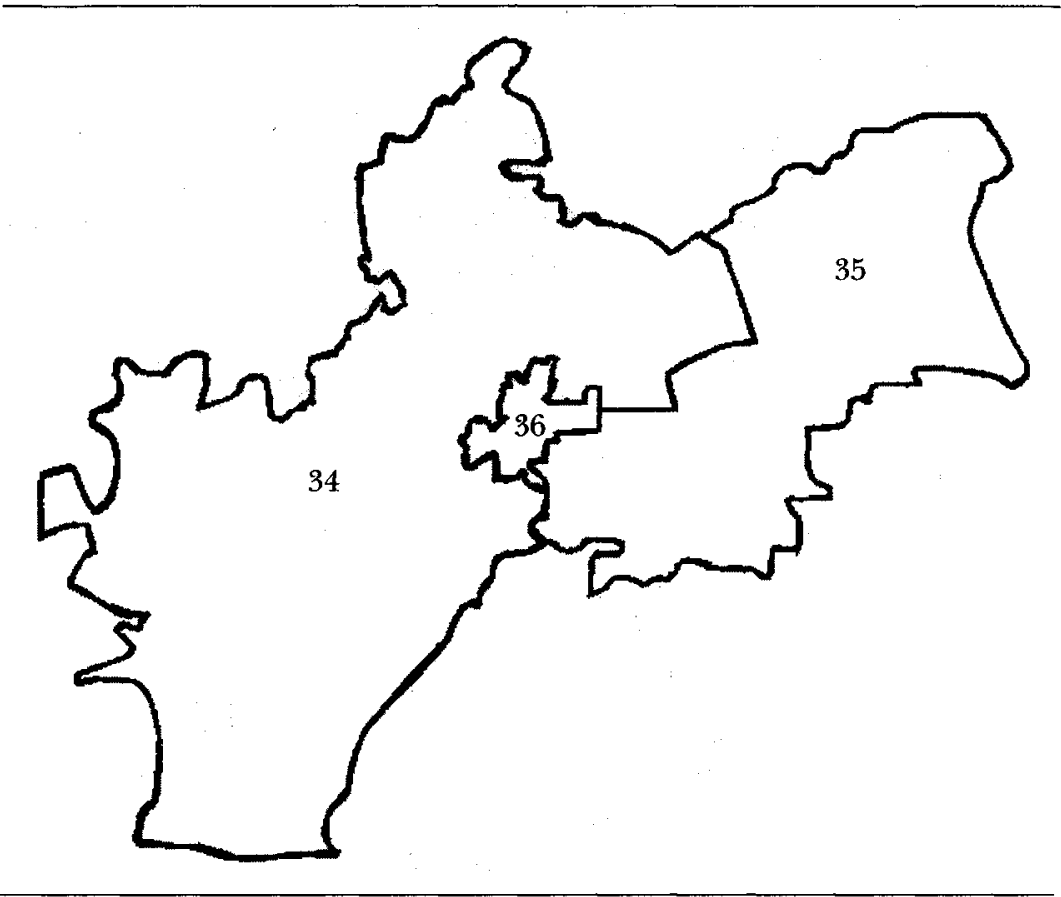

\section{Conclusiones}

El estudio de las implicaciones territoriales de los procesos electorales es necesario y particularmente apropiado cuando un proceso de reforma político-electoral se está llevando a cabo y poniendo a prueba. A fin de obtener una.justa representatividad en los congresos, es imprescindible idear métodos de distritación que den por resultado una razonable imparcialidad electoral. Éste fue el propósito principal del presente trabajo. De ahí que se incluyera, además de una revisión teórica de los principios de regionalización política para distritos electorales, un ejercicio práctico en donde se aplicaron un criterio y un algoritmo alternativos.

Indiscutiblemente, la distritación oficial de 1996 representó una mejora sustancial respecto a la de 1978 en muchos aspectos, tales co- 


\section{MAPA 3}

Redistritación experimental de la Zona Metropolitana de la Ciudad de México

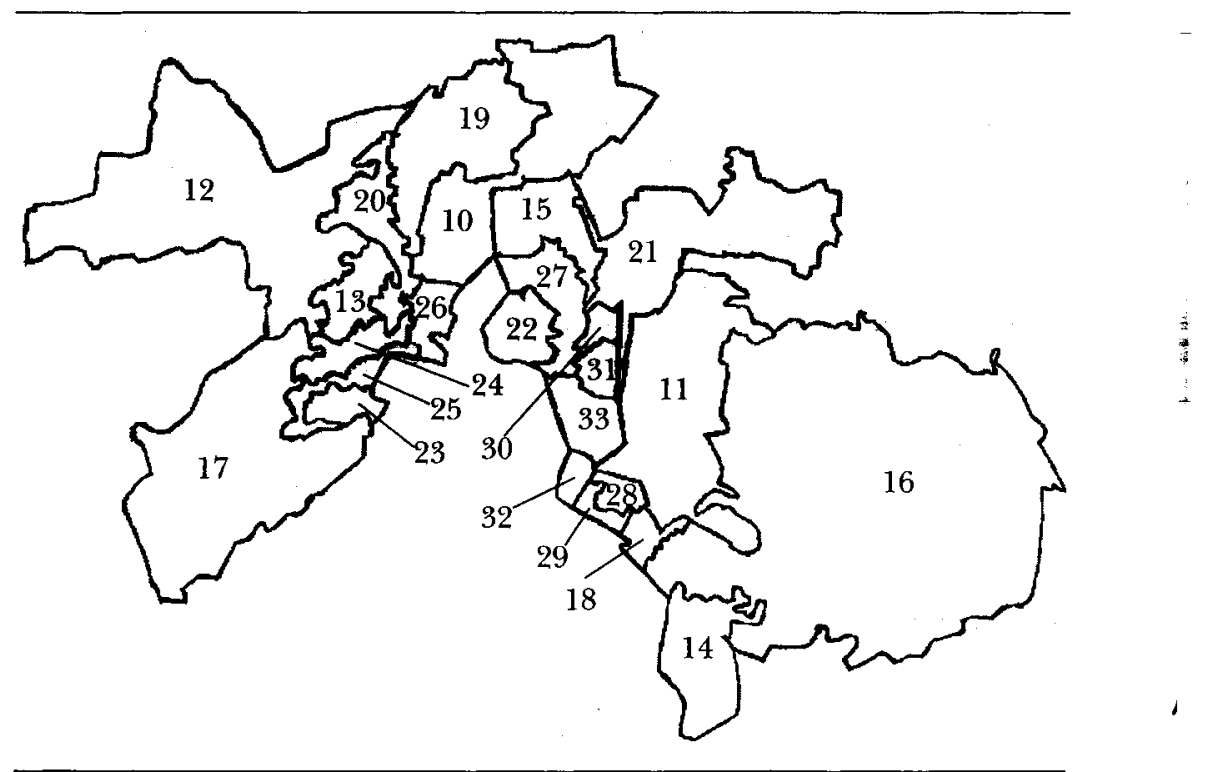

mo la actualización en el grado de representatividad ciudadana, la imparcialidad y la transparencia en el método. La mejor muestra de que la distritación vigente es imparcial son los resultados de las elecciones del 6 de julio de 1997 y del 2 de julio de 2000.

Sin embargo, vistas las mejoras logradas en el ejercicio anterior, es posible apreciar la factibilidad de perfeccionar los métodos de regionalización electoral a fin de lograr adelantos consistentes hacia una:justa geografía de la representación popular. El método utilizado en la distritación oficial vigente es perfectible. Se comprobó ampliamente la posibilidad de disminuir las diferencias poblacionales entre distritos; la razón fundamental de esta mejora fue el haber combinado sin distinción municipios y AGEB en el algoritmo. Además, la utilización de estas unidades geográficas se ciñe al mandato constitucional de utilizar población censal.

Debido a limitaciones técnicas y de tiempo, no fue posible abundar más en el ejercicio, especialmente en el punto de "vías de comunicación y accesibilidad", pero del avance logrado se desprende que 
la mejoría fue significativa, y el modelo no contradice la inserción de este criterio. La utilización del criterio de comunidad de interés al nivel metropolitano y no metropolitano, rindió un óptimo resultado en términos numéricos y pudo mantener el interés sobre la integridad de comunidades.

Se puede concluir entonces que los aspectos imperativos y deseables de este ejercicio alternativo, que eran 1) avanzar en el criterio de "comunidad de interés" y 2) reducir las diferencias poblacionales entre los distritos, pueden perfeccionarse en posteriores investigaciones. En especial, se debe poner atención tanto en el algoritmo como en las unidades geográficas que se utilizan.

En este sentido, es seguro que el contenido y la frecuencia de este tipo de estudios aumentarán conforme se consolide la práctica democrática y se convierta en un asunto rutinario más que en uno novedoso y de primera plana, como lo es en la actualidad.

\section{Bibliografía}

Aristóteles (1985), Ética nicomaquea, México, Porrúa.

Bourne, Larry S. y J. W. Simmons (1978), Systems of Cities Readings on Structure, Growth, and Policy, Nueva York, Oxford University.

Chorley, Richard y Peter Hagget (1967), Models in Geography, Londres, Methuen.

Donovan, B. (1991), "North Carolina Computer Draws some Labyrinthine Lines", Congressional Quarterly Weekly Report, vol. 49, núm. 28, pp. 1916-1918.

Elliot, Jeffrey H. y Ali Sheikh R. (1984), The Presidential Congressional Political Dictionany, Santa Bárbara, California, ABC-Clio Information Services.

Gelman, Andrew y Gary King (1994), "Enhancing Democracy through Legislative Redistricting", The American Political Science Review, vol. 88, núm. 3, pp. 541-559.

Grigg, David (1967), "Regions, Models, and Classes", en Chorley y Haggett (eds.), Models in Geography, Londres, Methuen, pp. 461-509.

Grofman, Bernard et al. (eds.) (1982), Representation and Redistricting Issues, Lexington, Lexington Books.

Instituto Federal Electoral (1996), La redistritación electoral mexicana de 1996: Informe sobre los resultados de la redistritación que presenta el Comité Técnico para el Seguimiento y Evaluación de la Redistritación al Consejo General del Instituto Federal Electoral, México, Registro Federal de Electores del IFE.

Johnston, Ronald J. (1979), Political, Electoral and Spatial Systems: An Essay in Political Geography, Oxford, Clarendon Press.

Lujambio Irazabal, Alonso José R. (1987), La proporcionalidad política del sistema electoral mexicano 1964-1985, México, Departamento de Ciencias Sociales, Instituto Tecnológico Autónomo de México. 
Orr, Douglas (1970), Congressional Redistricting: The North Carolina Experience, Chapel Hill, University of North Carolina.

Ostdiek, Donald (1995), "Congressional Redistricting and District Typologies", The Journal of Politics, vol. 57, núm. 2, pp. 533-543.

Overby, Marvin y Kenneth Cosgrove (1996), "Unintended Consequences? Racial Redistricting and the Representation of Minority Interests", The Journal of Politics, vol. 58, núm. 2, pp. 540-550.

Pacheco, Guadalupe, Ricardo Becerra y José Woldenberg (1997), "La nueva geografía electoral. Consecuencias de la redistritación", Etcétera, núm. 192, p. 28.

Sánchez, Joan-Eugeni (1992), Geografía politica, Madrid, Síntesis.

Sanguin, André-Louis (1981), Geografía política, Barcelona, Oikos-tau (versión castellana de Carme Ferrer y Jordi García Jacas). 